\title{
Spinal sensory radiculopathy due to Angiostrongylus cantonensis infection
}

\author{
Gillian Wood, Shane Delamont, Michael Whitby and Richard Boyle
}

Princess Alexandra Hospital, Brisbane, QLD, 4102, Australia.

\begin{abstract}
Summary: The most common cause of eosinophilic meningitis is the rat lung worm Angiostrongylus cantonensis, a parasite which is endemic in the South East Asian and Pacific regions. While the typical clinical presentation is that of meningitis associated with an eosinophilic pleocytosis, a 45 year old man presented with a radiculomyelopathy, associated with an eosinophilic pleocytosis and cerebrospinal fluid antibodies to $\boldsymbol{A}$. cantonensis but without signs or symptoms of meningitis. A worm was demonstrated on both computed tomographic myelography and magnetic resonance imaging scan of the spinal cord.
\end{abstract}

\section{Introduction}

Since 1945 there have been many reports of eosinophilic meningitis in South East Asia and the Pacific Islands. ${ }^{1,2}$ In the early 1960 s, the causal relationship with Angiostrongylus cantonensis was established. The occurrence of this worm in the Pacific Islands probably relates to the introduction of infected giant African snails Achatina fulica some 20 years earlier, ${ }^{3}$ as well as infection spread from imported rats to the local snails.

We describe a case of $A$. cantonensis infection which appears to have originated in Fiji where the disease has not previously been reported, which presented unusually with a dermatomal migration of paraesthesia without meningeal symptoms, and in which the worm was identified by magnetic resonance imaging and computed tomographic (CT) myelography.

\section{Case report}

A 45 year old Caucasian male was admitted for investigation of an 8-week history of paraesthesia and numbness affecting his right leg, initially in the S1 distribution. The symptoms of paraesthesia had progressed in a dermatomal distribution from $\mathrm{S} 1$ to $\mathrm{L} 1$ in the right leg, with progressive resolution of paraesthesia in the dermatomes initially infected. He had, however, noted a persisting subjective alteration in light touch sensation in the S1-L1 dermatomes. He had no associated muscle weakness, gait abnormality, bladder or bowel symptoms, sexual dysfunction, headache, fever, neck stiffness or malaise.

Correspondence: M. Whitby, F.R.A.C.P., F.R.C.P.A. Accepted: 9 July 1990
The patient was a contract driller who had been working on Viti Levu, the largest of the Fijian Islands, at the time of onset of his illness. He had been there for the preceding 16 weeks and prior to that had been in Indonesia. The patient had spent most of the preceding 25 years in Papua New Guinea, the Solomon Islands, Indonesia and Far North Queensland and had a past history of multiple episodes of malaria and of Type II diabetes mellitus, controlled by diet alone. $\mathrm{He}$ consumed up to $120 \mathrm{~g}$ of alcohol daily, smoked 40 cigarettes per day and had no family history of neurological disease. He denied ingestion of snails or prawns; however, his diet regularly included salads and other uncooked vegetables.

The only definite neurological deficit on examination was reduction of pin prick and light touch sensation in L2 distribution on the right, and some slight alteration in sensation in the sensory S1, L5, L4, L3 and L1 dermatomes. Muscle strength and tone, reflexes and posterior column function were all normal. There was no evidence of optic atrophy, papilloedema, diabetic retinal abnormalities or meningeal signs or symptoms.

Biochemical and haematological examination proved normal, as did electromyographic studies. However, lumbar CT myelography showed an anteriorly located left sided lesion extending over the length of the $L 1$ vertebra, but poorly delineated in its location (Figure 1). No abnormality was seen affecting the nerve roots or their exits from the canal. Magnetic resonance imaging (MRI) scanning confirmed the presence of a small filling defect extending from the middle of the body of $L 1$ to the L1/2 intervertebral disc. Lumbar cerebrospinal fluid (CSF) examination demonstrated $180 \mathrm{cells} / \mu \mathrm{l}$, (12\% neutrophils, $65 \%$ lymphocytes, $12 \%$ mononuclear cells and $11 \%$ eosinophils), with 528 red 


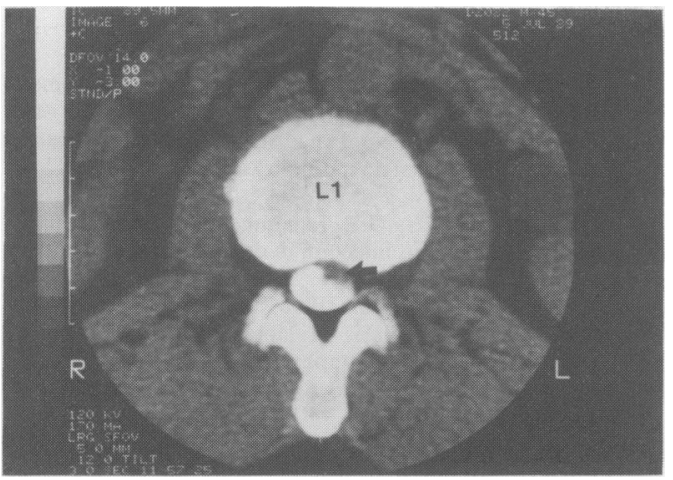

Figure 1 CT scan showing filling defect at level L1.

cells $/ \mu$ l. No organisms were seen or grown. CSF glucose was $3.3 \mathrm{mmol} / \mathrm{l}$ (reference range $2.1-3.7$ ), CSF protein $0.58 \mathrm{~g} / \mathrm{l}$ (reference range $0.15-0.45$ ). Cytological studies showed many lymphocytes, some eosinophils, together with monocytes and numerous red cells. No malignant cells were present nor were eggs, larvae or worms seen.

Enzyme-immunoassay of antibodies to soluble A. cantonensis antigen ${ }^{4}$ was performed on both the $\mathrm{CSF}$ and serum. In the CSF the reference ratio = 3.5 and in the serum $=20.0$ (Reference ratio $=$ optical density of the sample divided by that of the negative control. Reference range $>1=$ infection. Reference range $<1=$ no infection).

A diagnosis of $A$. cantonensis infection was made, and in view of the resolution of symptoms, no therapy offered. The patient returned to Far North Queensland and when reviewed by his neurologist 2 months later, the symptoms and signs had completely resolved.

\section{Discussion}

Angiostrongylus cantonensis is a rodent nematode which accidently infects man by ingestion of viable third stage larvae in uncooked or undercooked intermediate (slugs, snails) or transport hosts (freshwater prawns and crabs), or contaminated vegetables. The incubation period ranges from 1-16 days. ${ }^{1,2,5}$ The duration of the illness usually varies from 2-8 weeks; ${ }^{1,2,6}$ however, there have been reports of cases lasting 3-4 months. ${ }^{7}$

The classical clinical picture in man is that of a benign self-limiting meningitis with an eosinophilic pleocytosis. Headache occurs in $90 \%$ of patients and is the major symptom, while neck stiffness, fever and vomiting occur in about $50 \% .^{1,2}$ Paraesthesiae are quite common occurring in approximately $34-56 \%$ of many series. ${ }^{1,2}$ However, these are normally ill-defined, occurring asymmetrically, occasionally conforming to areas of dermatomal innervation on the extremities and less commonly on the face or trunk. They have been associated with marked hyperaesthesia or numbness and often persist for several weeks after recovery. ${ }^{1,2,6}$ In more severe forms of the disease, meningoencephalitis with altered conscious state may occur, ${ }^{2,6}$ but mortality is low, at less than $1 \%$.

The presentation in our patient is unusual in that he had a defined migratory paraesthesia in dermatomal distribution, without associated meningeal features. This presentation is more characteristic of that seen in dogs, where ascending paresis associated with lumbar hyperaesthesia occurs. ${ }^{8}$ These clinical symptoms are produced because the larvae in dogs preferentially migrate to the spinal cord rather than the brain. ${ }^{9}$ In addition, our case has some similarities to the 12 cases of $A$. cantonensis radiculomyeloencephalitis, reported by Klicks $e t$ $a l .^{5}$ where the patients developed severe lower limb paraesthesiae associated with a paresis and absent reflexes, but no sensory deficit. Autopsy of their one fatal case showed many worms in the spinal cord, but none in the brain or nerve roots.

CT myelogram and MRI recognition of a migratory Angiostrongylus worm (Figure 1) is unusual. Ko et al. ${ }^{10,11}$ reported probable lesions due to $A$. cantonensis in the brains of patients presenting with eosinophilic meningitis imaged by CT. The position of the worm in our patient suggests that the migratory dermatomal paraesthesia experienced was a reflection of the worm's migration up the spinal cord, affecting the sensory innervation of the dermatomes in which he described abnormal sensation. This suggests that the patient was infected with only one worm and this paucity of infecting organisms may account for the lack of classical meningeal and other systemic symptoms. Finally because of the relatively short incubation period of this disease (less than 16 days), we believe this patient must have acquired his infection in Fiji. It is known that Angiostrongylus cantonenis is present in the rat population of $\mathrm{Fiji}^{12}$ and cases of eosinophilic meningitis have been diagnosed (Dr Rajendra Parman, personal communication); to our knowledge, reports of eosinophilic meningitis from Fiji have not previously appeared in the literature.

\section{Acknowledgements}

We wish to thank Dr John Walker, Institute of Clinical Pathology and Medical Research, Westmead Hospital, Sydney for performing ELISA assays in this patient and Dr Paul Prociv, Department of Parasitology, University of Queensland for his helpful advice. 


\section{References}

1. Kuberski, T. \& Wallace, G.D. Clinical manifestations of eosinophilic meningitis due to Angiostrongylus cantonensis. Neurology 1979, 2: 1566-1570.

2. Punyagupta, S., Juttijudata, P. \& Bunnag, T. Eosinophilic meningitis in Thailand. Am J Trop Med Hyg 1975, 24: 921-931.

3. Alicata, J.E. The presence of Angiostrongylus cantonensis in Islands of the Indian Ocean and probable role of the giant African snail Achatina fulica in dispersal of the parasite to the Pacific Islands. Can J Zool 1966, 44: 1041-1049.

4. Cross, J.H. \& Chi, J.H.C. ELISA for the detection of Angiostrongylus cantonensis antibodies in patients with eosinophilic meningitis. South East Asian J Trop Med Pub Hlth 1982, 13: 73-76.

5. Kliks, M.M., Kroenke, K. \& Hardman, J.M. Eosinophilic radiculomyeloencephalitis: an Angiostrongyliasis outbreak in American Samoa related to ingestion of Achatina fulica snails. Am J Trop Med Hyg 1982, 31: 1114-1122.

6. Yii, C.Y. Clinical observations on eosinophilic meningitis and meningoencephalitis caused by Angiostrongylus cantonensis in Taiwan. Am J Trop Med Hyg 1976, 25: 233-249.

7. Reid, I.R. \& Wallic, W.E. The chronic and severe forms of eosinophilic meningitis. Aust NZ J Med 1984, 14: 163-165.

8. Mason, K.V. Canine neural angiostrongylus: the clinical and therapeutic features of 55 natural cases. Aust Vet J 1987, 64: 201-203.

9. Jindrak, K. \& Alicata, J.E. Experimentally induced Angiostrongylus cantonensis infection in dogs. Am J Vet Res 1970 , 31: 449-456.

10. Ko, R.C., Chiu, M.C., Kum, W. \& Chan, S.H. First report of human Angiostrongyliasis in Hong Kong diagnosed by computerized axial tomography (CAT) and enzyme linked immunosorbent assay. Trans $R$ Soc Trop Med Hyg 1984, 78: 354-355.

11. Ko, R.C., Chan, S.W., Chan, K.W. et al. Four documented cases of eosinophilic meningoencephalitis due to Angiostrongylus cantonensis in Hong Kong. Trans $R$ Soc Trop Med Hyg 1987, 81: 807-810.

12. Beaver, P.C., Jung, R.C. \& Cupp, E.W. (eds) Angiostrongylus cantonensis. In: Clinical Parasitology. Lea and Febiger: Philadelphia, USA, pp. 292-294. 